\title{
Supracerebellar infratentorial sitting craniotomy for a pinealoblastoma
}

\author{
Omar Choudhri, MD, ${ }^{1}$ and Steven D. Chang, MD² \\ 'Department of Neurosurgery, University of California, San Francisco; and \\ ${ }^{2}$ Stanford University Medical Center, Department of Neurosurgery, Stanford, California
}

\begin{abstract}
Pinealoblastomas are WHO grade IV tumors of the pineal region and comprise up to $50 \%$ of all pineal parenchymal tumors. They are highly aggressive tumors that spread along the craniospinal axis and are most commonly seen in children. The standard of care involves maximal surgical resection and chemoradiation following tissue diagnosis. We present the rare case of a large pinealoblastoma in an 18-year-old girl who presented with headaches and Parinaud's syndrome from tectal compression. An attempt was made at endoscopic transventricular biopsy of the tumor at an outside hospital, but it was aborted given bleeding at the biopsy site. We performed a supracerebellar infratentorial approach in a sitting position to achieve a gross-total resection of the tumor. This video case illustrates techniques for setting up a sitting craniotomy and approaching a previously biopsied hemorrhagic pinealoblastoma. The venous conglomerate at the tentorial incisura was found to be enveloped by the tumor and a thickened arachnoid scar. Surgical anatomy of the third ventricle and the pineal region is illustrated in this case through the process of surgical dissection and tumor resection.
\end{abstract}

The video can be found here: https://youtu.be/CzBOIFQ7Ayl.

KEYWORDS pinealoblastoma; sitting craniotomy; supracerebellar infratentorial; microsurgery; video 